\title{
Via de sinalização do Fator de Necrose Tumoral alfa (TNF-a), síntese e liberação no exercício físico
}

\author{
Signaling pathway of Tumor Necrosis Factor Alpha (TNF-a) release and \\ synthesis in exercise \\ Vía de señalización del Factor de Necrosis Tumoral Alfa (TNF-a), síntesis \\ y liberación en el ejercicio físico \\ Paulo Henrique BARBOSA ${ }^{1}$ \\ Flávia CARNEIRO ${ }^{1}$ \\ Anderson MARTELLI ${ }^{2}$ \\ Elke Lima TRIGO $\mathbf{T}^{3}$ \\ ${ }^{l}$ Graduação em Educação Física pela Faculdade FMG - Mogi Guaçu-SP; Especialista em Fisiologia do Exercício: Avaliação e \\ Prescrição do Treinamento, UNIFAE, São João da Boa Vista-SP, Brasil \\ ${ }^{2}$ Mestre em Ciências Biomédicas, Fundação Herminio Hometto - Uniararas. Docente do Curso de Graduação em Educação Física da \\ Faculdade Mogiana do Estado de São Paulo, FMG Mogi Guaçu - SP, Brasil \\ ${ }^{3}$ Mestre em Educação Física - EEFE-USP; Docente do Centro Universitário Senac, Brasil
}

\begin{abstract}
Resumo
Nos últimos anos inúmeros estudos tem retratado o Fator de Necrose Tumoral Alfa (TNF- $\alpha$ ) no exercício físico, sendo considerada uma citocina usualmente presente na resposta imunológica mediada por células. A presente revisão faz uma abordagem do TNF- $\alpha$, elucidando a sua via de sinalização e se a prática de exercícios físicos favorece a amenização desta citocina pró-inflamatória. Diante dos resultados, foi observado que o TNF- $\alpha$ é reconhecido como um mediador importante em muitos processos inflamatórios e foi possível verificar evidências de que o exercício físico pode atuar como um agente de respostas anti-inflamatórias reduzindo as concentrações de inúmeras citocinas pró-inflamatórias, dentre elas, o TNF- $\alpha$, podendo auxiliar no controle da inflamação crônica observado na obesidade. Ainda não está totalmente elucidada qual a melhor relação volume, intensidade e frequência de exercícios para potencializar os resultados.
\end{abstract}

Descritores: Fator de Necrose Tumoral alfa; Exercício; Inflamação.

\section{Abstract}

In recent years numerous studies have portrayed the Tumor Necrosis Factor Alpha (TNF- $\alpha$ ) in physical exercise, being considered a cytokine usually present in the cell-mediated immune response. The present review approaches TNF- $\alpha$, elucidating its signaling pathway and whether the practice of physical exercises favors the amelioration of this pro-inflammatory cytokine. In view of the results, it was observed that $\mathrm{TNF}-\alpha$ is recognized as an important mediator in many inflammatory processes and it was possible to verify evidence that physical exercise can act as an agent of anti-inflammatory responses reducing the concentrations of numerous proinflammatory cytokines, among them, TNF- $\alpha$, which may aid in the control of chronic inflammation observed in obesity. It is still not fully elucidated what the best volume, intensity and frequency of exercises ratio is in order to maximize results.

Descriptors: Tumor Necrosis Factor-alpha; Exercise; Inflammation.

\section{Resumen}

En los últimos años numerosos estudios han retratado el Factor de Necrosis Tumoral Alfa (TNF- $\alpha$ ) en el ejercicio físico, siendo considerada una citocina usualmente presente en la respuesta inmunológica mediada por células. La presente revisión hace un abordaje del TNF- $\alpha$, elucidando su vía de señalización y si la práctica de ejercicios físicos favorece la mitigación de esta citocina proinflamatoria. En los resultados se observó que el TNF- $\alpha$ es reconocido como un mediador importante en muchos procesos inflamatorios y fue posible verificar evidencias de que el ejercicio físico puede actuar como un agente de respuestas antiinflamatorias reduciendo las concentraciones de innumerables citocinas pro-inflamatorias, entre ellas, el TNF- $\alpha$, pudiendo auxiliar en el control de la inflamación crónica observada en la obesidad. Todavía no se ha dilucidado cuál es la mejor relación volumen, intensidad y frecuencia de ejercicios para potenciar los resultados.

Descriptores: Factor de Necrosis Tumoral alfa; Ejercicio; Inflamación.

\section{INTRODUÇÃO}

A obesidade tem sido muito investigada por vários especialistas da área da saúde, por ser considerada um grande problema de saúde no Brasil e no mundo ${ }^{1}$. Um estudo abrangente publicado no Periódico Lancet, o número de pessoas obesas aumentou de 857 milhões em 1980 para 2,1 bilhões de pessoas em 2013, sendo que mais da metade dos 671 milhões de pessoas com sobrepeso no mundo vive em 10 países (em ordem pelo número de obesos): EUA, China, Índia, Rússia, Brasil, México, Egito, Alemanha, Paquistão e Indonésia. $\mathrm{O}$ Brasil já está ocupando o $5^{\circ}$ lugar nesta lista ${ }^{1}$.

A definição para obesidade, dada pela Organização Mundial de Saúde, é o acúmulo excessivo ou anormal de gordura, índice de massa corporal (IMC) $\geq 30 \mathrm{~kg} / \mathrm{m}^{2}$ que representa risco à saúde ${ }^{2}$.

$\mathrm{O}$ tecido adiposo secreta inúmeras citocinas e proteínas de fase aguda, as quais de forma direta ou indireta influenciam na produção e deslocamento de elementos relacionados à inflamação ${ }^{3,4}$. Existem indícios demonstrando que a inflamação gerada por esse quadro pode ser por ação da resistência à insulina e outras divergências relacionadas à adiposidade, por exemplo, a hiperlipidemia e a síndrome metabólica 5 .

Speretta ${ }^{6}$ retrata que vários estudos demonstram que a hipertrofia dos adipócitos, especialmente nas vísceras, causa uma instabilidade na homeostase metabólica dos adipócitos, e consequentemente, favorecendo o aumento da geração de adipocinas, uma citocina pró-inflamatórias, como por exemplo, fator de necrose tumoral alfa (TNF- $\alpha$ ), e reduzindo a produção de citocinas anti-inflamatórias, como interleucina10 (IL-10), resultando em uma inflamação crônica de baixa intensidade.

As citocinas são classificadas como um grupo de proteínas de baixo peso molecular, que atuam na intercomunicação celular ${ }^{7}$. Essas moléculas são liberadas em decorrência de diferentes estímulos e interagem com receptores específicos regulando a função celular ${ }^{8}$ e estão intimamente relacionadas ao processo inflamatório. O TNF- $\alpha$, foi descoberto em 1975 por Carswell e colaboradores, sendo considerada uma das principais citocinas relacionadas aos processos inflamatórios e imunes, agindo em diferentes partes do corpo 9 .

Quando são consideradas medidas mitigadoras da obesidade, várias modalidades de exercício podem prover muitos benefícios na prevenção e na intervenção desse quadro, diminuindo o processo inflamatório crônico de baixa intensidade, ou seja níveis de TNF- $\alpha$ e outras adipocinas ${ }^{6}$. 
Deste modo, o objetivo deste trabalho foi realizar uma abordagem do TNF- $\alpha$, elucidando a sua via de sinalização e se as práticas de exercícios físicos favorecem a amenização de substancias pró-inflamatórias como esta citocina e o aumento das moléculas anti-inflamatórias.

\section{MATERIAL E MÉTODO}

Para a composição da presente revisão foi realizado um levantamento bibliográfico nas bases de dados SCIELO, Medline, Pubmed, Portal de Periódicos da Coordenação de Aperfeiçoamento de Pessoal de Nível Superior (CAPES) e bibliotecas institucionais de artigos científicos publicados até 2017, utilizando como descritores isolados ou em combinação: TNF- $\alpha$, exercícios, inflamação, assim como, consultas de livros acadêmicos para complementação das informações.

Para seleção do material, efetuaram-se três etapas conforme descrito por Martelli ${ }^{10}$. A primeira foi caracterizada pela pesquisa do material que compreendeu entre os meses de fevereiro/2017 a outubro de 2017 com a seleção de 58 trabalhos. A segunda, leitura dos títulos e resumos dos trabalhos, visando uma maior aproximação e conhecimento com o tema. Após essa seleção, buscaram-se os textos que se encontravam disponíveis na íntegra, totalizando 41 trabalhos, sendo estes, inclusos na revisão.

Como critérios de inclusão dos artigos, analisaram-se a procedência da revista e indexação, estudos que apresentassem dados referentes ao exercício físico e as vias de sinalização do TNF- $\alpha$ publicados entre os anos de 1993 até o mais atual 2017. Como critério de exclusão utilizou-se referência incompleta e informações desacreditadas, já que essa pesquisa visa revisar os conhecimentos atualizados sobre o tema.

\section{RESULTADOS E DISCUSSÃO}

\section{- TNF- $\alpha$ e via de sinalização}

Em 1975 Carswell et. al. descobriram o fator de transcrição tumoral alpha (TNF- $\alpha$ ) sendo classificada como determinante aos processos inflamatórios do sistema imunológico, atuando em diversas partes do corpo 9 .

Papo e Shai11 (2005) relatam que com a infecção de bactérias Gram-positivas e Gram-negativas, pode aumentar o número de citocinas através das ligações Toll-like receptors (TLRs), Lipopolissacarídeo (LPS) e ácido lipoteicoico, podendo ter a liberação das moléculas da parede celular. O LPS pode causar febre pela produção de citocinas e pelo aumento da síntese de prostaglandinas nas células hipotalâmicas, sendo que ainda não descobriram o exato papel da febre, mais ela reduz o risco de piora das infecções e lesões ${ }^{12}$.

Neste sentido, o TNF- $\alpha$ possui diversas funções imunológicas ${ }^{9,13}$. É sintetizado por macrófagos durante canceres, infecções, doenças, caquexia e promove necrose tumoral. Esta citocina pode ser pró-inflamatória que inibe a proliferação de tumores promovendo a apoptose ${ }^{13}$. Segundo Weisberg e colaboradores ${ }^{14}$ em condições fisiológicas normais os adipócitos produzem TNF- $\alpha$, que é chamado de adipocina e no músculo é chamado de miocina.

Após sua síntese e liberação, o TNF- $\alpha$ irá ligar-se a receptores específicos denominados de receptores de TNF (TNF-R) I e II, para que possa produzir o seu efeito biológico $^{9}$. O TNF-RI recruta uma proteína que é ativadora das capazes e dispara a apoptose, sendo assim o TNF- $\alpha$ pode proliferar com a expressão de gene ou morte celular, mas alguns podem fazer as duas formas ${ }^{12}$. Esses receptores são membros da superfamília de receptor de TNF e levam ao recrutamento de proteínas de fatores associados ao receptor de TNF (TRAFS) para o citoplasma, que por sua vez ativam o fator nuclear kappa $B(\mathrm{NF}-\mathrm{kB})^{12}$.

$\mathrm{O}$ NF-kB em um conjunto de cinco subunidades diferentes: p65 ou Rel 11 A, Rel B, p52 e p50 promove a caquexia e hipotrofia muscular, porque além de ser expresso no tecido adiposo, também é expresso no músculo estriado esquelético, estando também envolvido no mecanismo de atrofia muscular pelo sedentarismo ${ }^{15}$.

A cascata de sinalização do TNF- $\alpha$ tem início por meio da inibição do complexo NF-kB, que está inativado no citoplasma da célula, ligando-se com I $\mathrm{B}$-quinase (IKK $\beta$ ), que contém IKK- $\alpha$ e IKK- $\beta$. A proteína inibitória IkB- $\alpha$ é fosforilada, ubiquitinada e degradada por proteassomas, liberando NF-к B para translocar para o núcleo ativando o Muscle Ring Finger-1 (MuRF-1) ${ }^{16,17}$. A via de sinalização do TNF- $\alpha$ é mostrada na Figura 1.

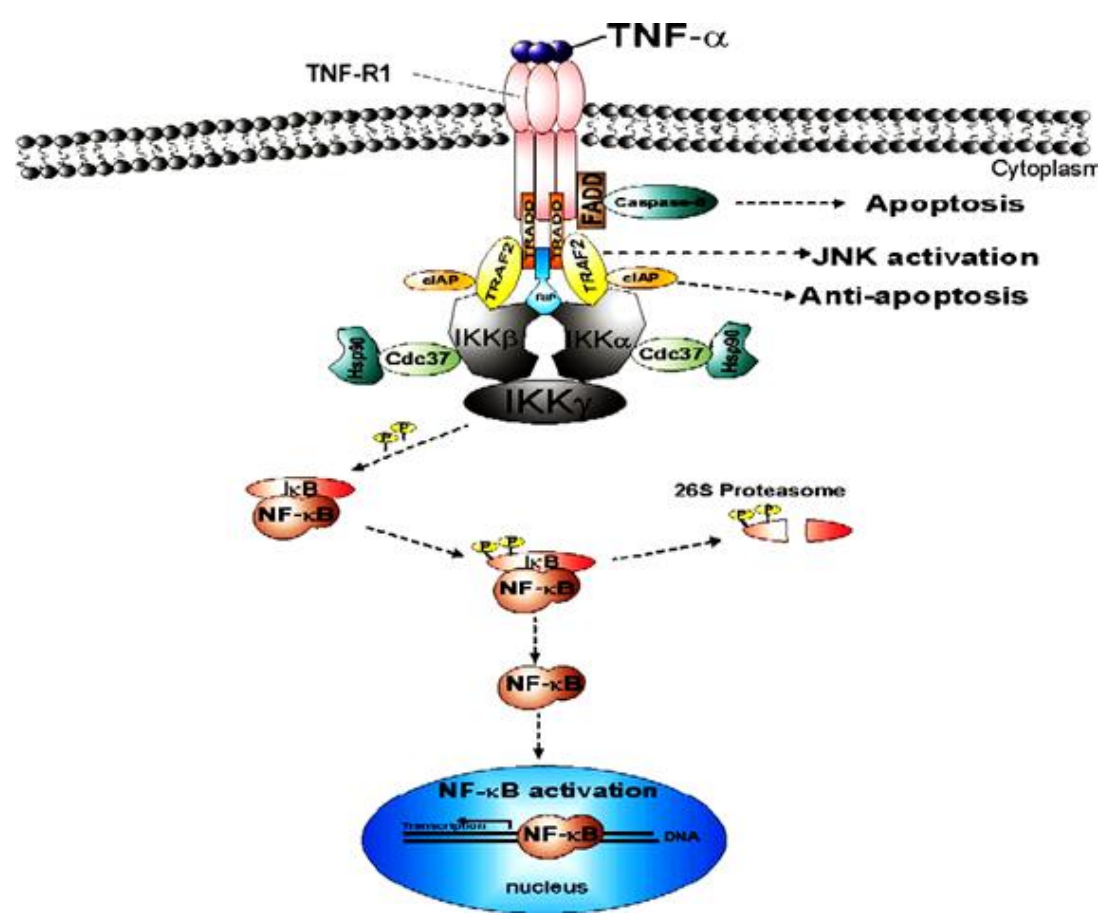

Figura 1: Via de sinalização TNF- $\alpha$. Extraído de Dirks-Naylor e Leeuwenburgh $^{18}$.

Fortin et al. ${ }^{19}$ descrevem que o TNF- $\alpha$ e algumas citocinas pró-inflamatórias, promovem supressão da via IGF1-Akt e resistência à insulina. Com a ativação da via AKT a via NFkB é minimizada, desta forma, inibindo a AKT, é observado uma contribuição significativa para hipotrofia muscular. Estudos recentes apontam para via da c Jun Nterminal Kinase (JNK) sendo responsável por fosforilar e inibir o substrato receptor da insulina (IRS) com a ativação da JNK pelo TNF- $\alpha{ }^{20}$.

Grivicich et al. ${ }^{21}$ relatam em seu estudo que as proteínas inibidoras da apoptose ou (IAP) exercem essa função inibindo as caspases que articulam o fator de transcrição NF-kB. O receptor de morte resulta no recrutamento do Fas a proteína associada com o domínio da morte (FADD), que recruta a caspase- 8 , com a ativação desta proteína, ela propaga um sinal apoptótico, promovendo apoptose ou morte celular programada ${ }^{22}$.

O TNF- $\alpha$ e exercícios fisicos

Fazendo uma correlação do TNF- $\alpha$ com exercícios, Phillips et al. ${ }^{23}$ treinaram mulheres idosas na musculação, 3 vezes na semana durante 10 semanas, com intensidade de $80 \%$ e 1 repetição máxima (RM) e analisaram que os níveis das adipocinas inflamatórias, TNF- $\alpha$ e IL-6 reduziram significativamente.

Um estudo envolvendo 14 adolescentes obesos submetidos a treino em musculação a $65-85 \%$ de $1 \mathrm{RM}, 3$ vezes na semana em dias não consecutivos, com sessão de 60 minutos, durante 16 semanas, foi verificado que houve uma redução do percentual de gordura e redução dos níveis de inflamação sistêmica com diminuição dos níveis de TNF- $\alpha$ e IL- $6^{24}$.

Córdova e et al..$^{25}$ dividiram 54 idosas sedentárias em dois grupos, grupo de treino resistido de musculação e grupo controle, sendo submetido esses grupos a exercícios com intensidade de $70 \%$ de 1 RM, 3 vezes por semana, em dias 
não consecutivos, com 50 minutos cada sessão de treinamento com 9 exercícios. Eles concluíram que o grupo de treinamento resistido apresentaram níveis consideravelmente menores de TNF- $\alpha$, IL-6, Interferon (IFN-y) e pressão arterial sistólica comparado com o grupo controle.

Borges e Carvalho ${ }^{26}$, em um hospital universitário investigaram 75 pacientes com doença pulmonar obstrutiva crônica (DPOC), sendo que, 46 do grupo controle e 29 do grupo de treinamento, com exercícios a $80 \%$ de $1 \mathrm{RM}$. Os pacientes foram avaliados no segundo dia de internação, na alta e 30 dias após a alta. O grupo controle apresentou uma redução de força nos membros inferiores, em contrapartida o grupo de treinamento aumentou a força nos membros inferiores, sendo que os flexores de quadril aumentaram em $17,1 \%$, e dos flexores de joelho em $16,2 \% \%$ com uma queda considerável nos níveis de marcadores de inflamação - TNF$\alpha$, IL-6 e IL-8. Estudos apontam que as citocinas têm como alvo restaurar o tecido lesionado pós treino ${ }^{27,28,29}$. Neste caso, o TNF- $\alpha$ e a IL-6, exercem funções na restauração do tecido lesionado ${ }^{30}$.

Tomoreli et al. ${ }^{31}$ fizeram um estudo randomizado com 38 idosas obesas, sendo que 19 grupo controle, sem realizar nenhum exercício e 19 no grupo de treinamento sendo aplicado 8 semanas de treinamento. $\mathrm{O}$ treino foi composto de 8 exercícios de 3 séries de 10-15 RM realizados 3 vezes na semana, sem nenhuma intervenção nutricional. A antropometria foi feita por DEXA, foram feitos testes de 1 $\mathrm{RM}$, e exames de sangue pré e pós treinamento. Os resultados demonstraram que o treino resistido reduziu a gordura corporal e aumentou a massa muscular, promovendo o aumento de força e verificado redução dos marcadores da inflamação, TNF- $\alpha$, proteína C-reativa (PCR) e IL-6. Este estudo também mostrou uma melhora no perfil lipídico com aumento do HDL-colesterol e redução do LDL-colesterol e na glicemia em jejum.

Foram randomizados 17 adultos obesos para fazer treino intervalado de alta intensidade (HIIT) ou treinamento contínuo de intensidade moderada (MICT), depois de completar 12 sessões de treino no período de 3 semanas, os participantes poderiam escolher treinar de forma independente HIIT ou MICT por 30 minutos, 4 dias na semana, com duração de 5 semanas, sendo que os resultados incluíram aptidão cardiorrespiratória $\left(\mathrm{VO}_{2}\right.$ pico), lipídios, marcadores inflamatórios, e os exercícios foram medidos pela escada de prazer de atividade física validada. $\mathrm{Na}$ aderência e no prazer não houve resultados significativos, mas no HIIT houve uma diminuição nos níveis de colesterol de lipoproteínas de baixa intensidade e aumento do $\mathrm{VO}_{2}$ pico em relação ao MICT. A proteína IL-6 e PCR aumentaram no HIIT e diminuiu no MICT, ou seja, o HIIT teve uma associação com aumento desses marcadores na fase aguda. Neste estudo, foi sugerido a inclusão de um grupo controle para comparação dos dados ${ }^{32}$.

Steckling ${ }^{33}$ estudou mulheres com síndrome metabólica (SM), o treino foi de 3 vezes na semana, com duração de 12 semanas e mais 2 de destreinamento, sendo HIIT na esteira com intensidade individualizada e acompanhada por monitores cardíacos. Após o período de treino, os níveis de nitrito e nitrato (NOx) aumentaram e conservaram no período de destreinamento. Porém os níveis de IL-1, IL-6, TNF- $\alpha$, IFN-y, apresentaram uma considerável redução, do mesmo modo que retornaram ao normal despois de duas semanas de destreinamento.

Uma pesquisa realizada com portadores de Diabetes Mellitus do tipo 2 e um grupo controle, com apenas 1 treino agudo de HIIT, sendo 7 tiros com 1 minuto de intervalo em um ciclo ergômetro com intensidade de $85 \%$. Esta sessão teve efeito anti-inflamatório com níveis mais baixos de expressão de proteínas de superfície de receptor TLR2 e TNF- $\alpha^{34}$.

Estudo randomizado foi conduzido por Allen et al. ${ }^{35}$ com 54 adultos sedentários divididos em 3 grupos, HIIT, Treinamento de sprint intermitente prolongado (PIST), e um grupo controle. O treino foi de 3 vezes na semanas, durante 9 semanas em um bicicleta ergométrica, com analise pré e pós das medidas de antropometria, $\mathrm{VO}_{2}$ pico e coleta de sangue para analisar PCR e TNF- $\alpha$. Ambos os treinos aumentaram o $\mathrm{VO}_{2}$ pico, mas no HIIT foi observado redução da circunferência na altura da cintura em relação ao grupo controle, e os marcadores de inflamação não houve diferenças entre os grupos. Bartlett et al. ${ }^{36}$ compararam os efeitos do HIIT e MICT em 10 semanas de treinamento e também não observaram diferenças nos marcadores de inflamação sistêmica com o treino.

Em 2017 Cypiran et al. $^{37}$ avaliaram atletas, todos submetidos a três sessões de exercícios, HIIT curto com intervalo de trabalho de 30 segundos, HIIT longo com intervalo de trabalho de 3 minutos e exercício de carga constante. Os resultados revelaram que os marcadores de regulação autonômica cardíaca, inflamação e dano muscular não revelaram diferenças consideráveis entre atletas de resistência e sprint.

Quando o exercício é intenso, se associa ao desenvolvimento da incidência de doenças infecciosas, principalmente nas vias aéreas superiores (IVAS), isso é muito estudado nos esportes de alto desempenho ${ }^{38}$. Segundo Todo-Bom e Pinto ${ }^{39}$ em praticantes dos exercícios moderados as infecções respiratórias são menos frequentes, porém atletas que praticam exercícios de grande intensidade e de grande duração estão sujeitos a infecções. Assim, recomenda-se que as funções do sistema imune e infecções respiratórias sejam interpretadas por uma curva em "J", onde os praticantes de exercícios moderados apresentam riscos mais baixos de problemas com infecção e os de alta intensidade estão sujeitos a esses quadros ${ }^{40}$.

Scherr et al. ${ }^{41}$ avaliaram 105 maratonistas de $42 \mathrm{~km} \mathrm{e}$ observaram um aumento considerável de IL-6, IL-10 e de TNF- $\alpha$, porém esses níveis baixaram e se normalizaram depois de 72 horas, evidenciando um pico de citocinas posteriormente aos exercícios intensos e prolongados.

\section{CONSIDERACÕES FINAIS}

O exercício físico é uma pratica não medicamentosa eficaz contra obesidade, considerada um problema de saúde pública mundial e um quadro que favorece uma inflamação crônica de baixa intensidade. Neste estudo foi possível verificar evidências de que o exercício físico pode atuar como um agente de respostas anti-inflamatórias reduzindo as concentrações de inúmeras citocinas pró-inflamatórias, dentre elas, o TNF- $\alpha$, podendo auxiliar no controle dessa inflamação crônica observado na obesidade, assim como reduzir riscos de outras doenças crônicas não transmissíveis como diabetes, hipertensão arterial e aterosclerose, porém, ainda não está totalmente elucidada qual a melhor relação volume, intensidade e frequência de exercícios para potencializar os resultados. Portanto, novos estudos devem ser realizados para um maior aprofundamento das vias de sinalização do TNF- $\alpha$ e sua associação ao exercício físico.

\section{REFERÊNCIAS}

1. Ng M, Fleming T, Robinson M, Thomson B, Graetz N, Margono $\mathrm{C}$ et al. Global, regional, and national prevalence of overweight and obesity in children and adults during 1980-2013: a systematic analysis for the Global Burden of Disease Study 2013. Lancet. 2014; 384(9945):766-81.

2. World Health Organization. Obesity: preventing and managing the global epidemic. Report of a WHO consultation. 2000:1-253.

3. Bulló M, Garcia-Lorda P, Megias I, Salas-Salvadó J. Systemic inflammation, adipose tissue tumor necrosis 
factor, and leptin expression. Obes Res. 2003 11(4):525-31.

4. Trayhurn P. Adipocyte biology. Obes Rev. 2007; 8(Suppl1):41-4.

5. Yudkin JS, Stehouwer CD, Emeis JJ, Coppack SW. Creactive protein in healthy subjects: associations with obesity, insulin resistance, and endothelial dysfunction: a potential role for cytokines originating from adipose tissue? Arterioscler Thromb Vasc Biol. 1999; 19(4):972-8.

6. Speretta GFF, Leite RD, Duarte ACG. O. Obesidade, inflamação e exercício: foco sobre o TNF-alfa e IL-10. Revista HUPE. 2014; 13(1):61-9.

7. Abbas AK, Lichtman AH, Pober JS. Citocinas. In: Imunologia celular e molecular. 2. ed. Rio de Janeiro: Revinter; 1998. p.253-76.

8. Hamblin AS. Cytokines and cytokine receptors. 2. ed New York: Oxford University Press; 1993.

9. Vitale RF, Ribeiro FAQR. O papel do Fator de Necrose Tumoral Alfa (TNF- $\alpha)$ no processo de erosão óssea presente no colesteatoma adquirido da orelha média. Rev Bras Otorrinolaringol. 2007; 73(1):123-7.

10. Martelli A. Potencial da prática de exercícios físicos regulares como método não farmacológico no controle da Hipertensão Arterial Sistêmica. Rev Desenv Pessoal. 2013; 3:39-51.

11. Papo N, Shai Y. A molecular mechanism for lipopolysaccharide protection of Gram-negative bacteria from antimicrobial peptides. J Biol Chem. 2005; 280(11):10378-87.

12. Abbas AK, Lishtman AH, Pillai S. Imunologia celular e molecular. 8. ed. Rio de Janeiro: Elsevier; 2015.

13. Rose DP, Komninou D, Stephenson GD. Obesity, adipocytokines, and insulin resistance in breast cancer Obes Rev. 2004;5(3):153-65.

14. Weisberg SP, Mccann D, Desai M, Rosenbaum M, Leibel RL, Ferrante AW Jr. Obesity is associated with macrophage accumulation in adipose tissue. J Clin Invest 2003; 112(12):1796-808.

15. Lima WP. Mecanismos moleculares associados à hipertrofia e hipotrofia muscular: relação com a prática do exercício físico. Rev Bras Fisiologia do Exercício. 2017;16(2):95-113.

16. Yang J, Park Y, Zhang H, Xu X, Laine GA, Dellsperger $\mathrm{KC}$ et al. Feed-forward signaling of TNF- $\alpha$ and NF- $\kappa B$ via IKK- $\beta$ pathway contributes to insulin resistance and coronary arteriolar dysfunction in type 2 diabetic mice. Am J Physiol Heart Circ Physiol. 2009;296(6): H1850-8.

17. Peterson JM, Bakkar N, Guttridge DC. NF-KB signaling in skeletal muscle health and disease. Curr Top Dev Biol. 2011;96:85-119.

18. Dirks AJ, Leeuwenburgh C. Tumor necrosis factor alpha signaling in skeletal muscle: Effects of age and caloric restriction. J Nutr Biochem. 2006; 17(8):501-8.

19. Fortin SP, Ennis MJ, Savitch BA, Carpentieri D, McDonough WS, Winkles JA et al. Tumor Necrosis Factor-Like weak inducer of apoptosis stimulation of glioma cell survival is dependent on Akt2 function. Mol Cancer Res. 2009;7(11):1871-81.

20. Hotamisligil GS. Inflammation and metabolic disorders Nature. 2006; 444(7121):860-7.

21. Grivicich I, Regner A, Rocha AB. Morte Celular por Apoptose. Rev Bras Cancerol. 2007; 53(3):335-43.

22. Kreuz S, Siegmund D, Rumpf JJ, Samel D, Leverkus M, Janssen $\mathrm{O}$ et al. NFKB activation by Fas is mediated through FADD, caspase-8, and RIP and is inhibited by FLIP. J Cell Biol. 2004;166(3):369-80

23. Phillips M, Flynn MG, Mcfarlin BK, et al. Resistance training at eight-repetition maximum reduces the inflammatory milieu in elderly women. Med Sci Sports Exerc. 2010; 42(2):314-25.

24. Shultz SP, Dahiya R, Leong GM, et al. Muscular strength, aerobic capacity, and adipocytokines in obese youth after resistance training: a pilot study. Australas Med J. 2015; 8(4):113-20.

25. Córdova C, Lopes-e-Silva F Jr, Pires AS, Souza VC, Brito CJ, Moraes $\mathrm{CF}$ et al. Long-term resistance training is associated with reduced circulating levels of IL-6, IFN$\mathrm{y}$ and TNF- $\alpha$ in elderly women. Karger. 2011; 18(3):165-70

26. Borges RC, Carvalho CR. Impact of resistance training in chronic obstructive pulmonary disease patients during periods of acute exacerbation. Arch Phys Med Rehabil. 2014;16(9):1638-45.

27. Peake JM, Nosaka K, Suzuki K. Characterization of infl ammatory responses to eccentric exercise in humans. Exerc Immunol Rev. 2005; 11:64-85.

28. Tidball JG. Inflammatory processes in muscle injury and repair. Am J Physiol Regul Integr Comp Physiol. 2005; 288(2):R345-53.

29. Pedersen BK, Febbraio MA. Muscle as an endocrine organ: focus on muscle-derived interleukin-6. Physiol Rev. 2008; 88(4):1379-406.

30. Smith LL. Cytokine hypothesis of overtraining: a physiological adaptation to excessive stress? Med Sci Sports Exerc. 2000; 32(2):317-31.

31. Tomeleri CM, Ribeiro AS, Souza MF, Schiavoni D, Schoenfeld BJ, Venturini D et al. Resistance training improves inflammatory level, lipid and glycemic profiles in obese older women: A randomized controlled trial. Exp Gerontol. 2016; 84:80-7.

32. Vella CA, Taylor K, Drummer D. High-intensity interval and moderate-intensity continuous training elicit similar enjoyment and adherence levels in overweight and obese adults. Eur J Sport Sci. 2017;17(9):1203-11.

33. Steckling FM. Efeitos do treinamento no perfil oxidativoinflamatório de mulheres com síndrome metabólica [dissertação]. Santa Maria: Universidade Federal de Santa Maria, Centro de Educação física e Desportos, 2015.

34. Durrer C, Francois M, Neudorf H, Little JP. Acute HIIT reduces TLR2 expression in type 2 diabetes. Am J Physiol Regul Integr Comp Physiol. 2017; 312(4):R529-38.

35. Allen NG, Higham SM, Mendham AE, Kastelein TE, Larsen PS, Duffield, R. The effect of high-intensity aerobic interval training on markers of systemic inflammation in sedentary populations. Eur J Appl Physiol. 2017; 117(6):1249-56.

36. Bartlett DB, Shepherd SO, Wilson OJ, Adlan AM, Wagenmakers AJM et al. Neutrophil and monocyte bactericidal responses to 10 weeks of low-volume highintensity interval or moderate-intensity continuous training in sedentary adults. Oxid Med Cell Longev. 2017; 2017:8148742

37. Cipryan L, Tschakert G, Hofmann, P. Acute and postexercise physiological responses to high-intensity interval training in endurance and sprint athletes. J Sports Sci Med. 2017; 16(2):219-29.

38. Peters EM. Exercise, immunology and upper respiratory tract infections. Int J Sports Med. 1997; 18(Suppl 1):S69-77.

39. Todo-Bom A, Pinto AM. Exercício físico - Resposta imunoinflamatória. Rev Port Imunoalergologia. 2007; 15(2):123-33.

40. Nieman DC. Exercise, infection and immunity. Int $\mathbf{J}$ Sports Med. 1994;15(Suppl 3):S131-141.

41. Scherr J, Braun S, Schuster T, Hartmann C, Moehlenkamp S, Wolfarth B et al. 72-h kinetics of high- 
sensitive troponin $\mathrm{T}$ and inflammatory markers after marathon. Med Sci Sports Exerc. 2011;43(10):1819-27.

\section{CONFLITO DE INTERESSES}

Os autores declaram não haver conflitos de interesse.

\section{AUTOR PARA CORRESPONDÊNCIA}

Anderson Martelli

martellibio@hotmail.com

Submetido em 23/11/2017

Aceito em 09/01/2018 